\title{
Associated production of photons and other gauge bosons at CMS
}

Joshua Kunkle, on behalf of the CMS Collaboration*

University of Maryland (US)

E-mail: jmkunkle@gmail.com

\begin{abstract}
We present studies of diboson and triboson production in pp collisions based on data recorded by the CMS detector at the LHC. These include precise measurements the production cross section of these processes at center-of-mass energies of 8 and $13 \mathrm{TeV}$. Final states where one or more photons are produced in association of $\mathrm{W}$ and $\mathrm{Z}$ bosons, reconstructed through their decay products, are used. The results are interpreted in terms of constraints on anomalous triple and quartic gauge couplings.
\end{abstract}

38th International Conference on High Energy Physics 3-10 August 2016

Chicago, USA

${ }^{*}$ Speaker. 


\section{Introduction}

Multiboson final states provide a broad perspective on the Standard Model. Final states including photons - the focus of this note - are unique probes of both the Standard Model and new physics. Photons participate in the electroweak sector, but unlike the $\mathrm{W}$ and $\mathrm{Z}$ bosons, photons provide clean experimental signatures without incurring reductions in the signal from additional branching fractions. Precision cross section measurements are made using high-rate multiboson processes. In addition, multiboson final states access very rare standard model processes In multiboson final states that are newly accessible with the large integrated luminosity of the LHC. The higher center-of-mass energy as well as the integrated luminosity of the LHC also allow stringent limits to be placed on new physics that could appear as anomalous modifications of gauge couplings. A summary of CMS measurements of these processes as well as others can be found here ${ }^{1}$.

\section{Effective field theory for anomalous gauge couplings}

If the energy scale of new physics is just beyond the reach of the LHC such that new particles cannot be produced as a resonance, effects from the new physics may appear in the high energy tails of standard model processes. Such effects are parameterized using an effective field theory [1]. The effective field theory, which is analogous to Fermi's theory [2], extends the standard model Lagrangian to higher order terms that are suppressed by the energy scale of new physics. The Lagrangian takes the form,

$$
\mathscr{L}_{N P}=\mathscr{L}_{S M}^{4}+\frac{1}{\Lambda^{2}} \mathscr{L}^{6}+\frac{1}{\Lambda^{4}} \mathscr{L}^{8}+\cdots
$$

The terms having odd powers are generally dropped because they lead to other significant modifications to standard model physics such as lepton and baryon number violation [1]. The dimension-six term leads to anomalous triple gauge couplings (aTGC) and tight limits on those terms can be placed using final states involving triple gauge couplings. The dimension-eight term leads to modifications of both quartic and triple gauge couplings. However, final states that are directly sensitive to triple gauge couplings place much stronger limits on aTGC. Therefore, limits are placed on anomalous quartic gauge couplings (aQGC) using final states containing quartic couplings assuming that no aTGC exist. From a theoretical perspective, new physics effects are expected to appear in the dimension-six term, but in some theories may also appear more dominantly in the dimension-eight term [3]. In some cases, in particular for anomalous neutral couplings, a vertex formalism is used.

\section{Results from CMS}

The CMS experiment [4] has produced a number of multiboson results involving photons. In this note, some of the more recent and pertinent results are discussed.

The measurement of $\mathrm{Z} \gamma$ in the final state where the $\mathrm{Z}$ boson decays to neutrinos has been performed both at $8 \mathrm{TeV}$ using $19.6 \mathrm{fb}^{-1}$ of data [5] and at $13 \mathrm{TeV}$ using $2.3 \mathrm{fb}^{-1}$ of data [6]. This

\footnotetext{
${ }^{1}$ https://twiki.cern.ch/twiki/bin/view/CMSPublic/PhysicsResultsCombined
} 
final state takes advantage of the larger branching fraction of the $\mathrm{Z}$ boson to neutrinos relative to the branching fraction to charged leptons resulting improved sensitivity to anomalous gauge couplings.

Candidate events are triggered by a single high $\mathrm{E}_{\mathrm{T}}$ photon. The photon is required to have $\mathrm{E}_{\mathrm{T}}>$ $145 \mathrm{GeV}$ for the $8 \mathrm{TeV}$ analysis and $\mathrm{E}_{\mathrm{T}}>175 \mathrm{GeV}$ for the $13 \mathrm{TeV}$ analysis. The difference results from the increase in the trigger threshold which was made account for the higher instantaneous luminosity provided by the LHC during $13 \mathrm{TeV}$ data taking. Additional shower shape and isolation requirements are imposed on the photon to reduce the contamination from hadronic jets. The transverse momentum imbalance $\left(\mathrm{E}_{\mathrm{T}}^{\mathrm{miss}}\right)$ is required to be large in magnitude and be well separated from the photon in $\phi$. In the $13 \mathrm{TeV}$ result the magnitude of $\mathrm{E}_{\mathrm{T}}^{\mathrm{miss}}$ is required to be greater than 170 $\mathrm{GeV}$ while in the $8 \mathrm{TeV}$ analysis a minimized $\chi^{2}$ method is used calculate a related quantity that improves the resolution of fake $\mathrm{E}_{\mathrm{T}}^{\text {miss }}$ which is required to be greater than $120 \mathrm{GeV}$. Events are also removed if they contain any identified jets, electrons, or muons.

Backgrounds to the signal process consist of $\mathrm{W} \gamma$ events where the $\mathrm{W}$ boson decays to a lepton which is subsequently not reconstructed, events where the photon is a misidentified electron or jet, and instrumental backgrounds that can produce energy deposits that mimic a photon. The $W \gamma$ background is estimated using Monte Carlo simulation samples while the remaining backgrounds are determined using data-driven methods.

Figure 1 shows the photon $E_{T}$ distributions for the $8 \mathrm{TeV}$ and $13 \mathrm{TeV}$ results. The signal is clearly observed above the predicted background. The measured cross sections agree well with the theoretical predictions which are shown in Table 1. Strong limits on anomalous neutral gauge couplings are set using the $8 \mathrm{TeV}$ analysis.

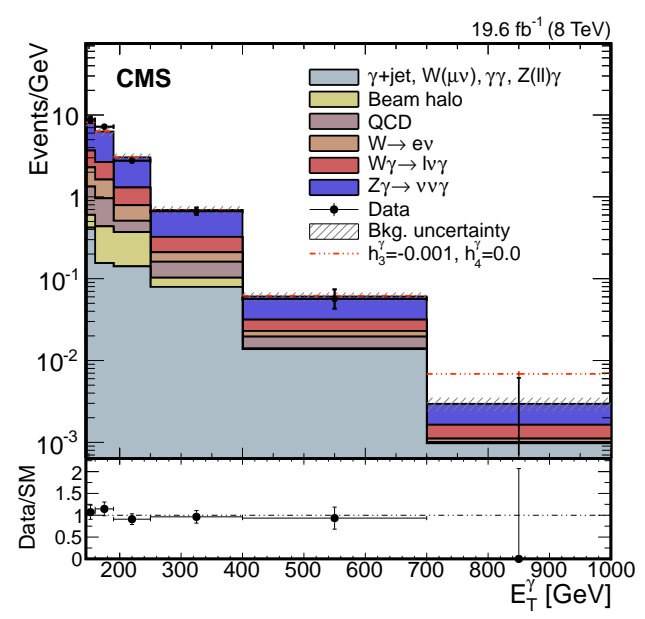

(a)

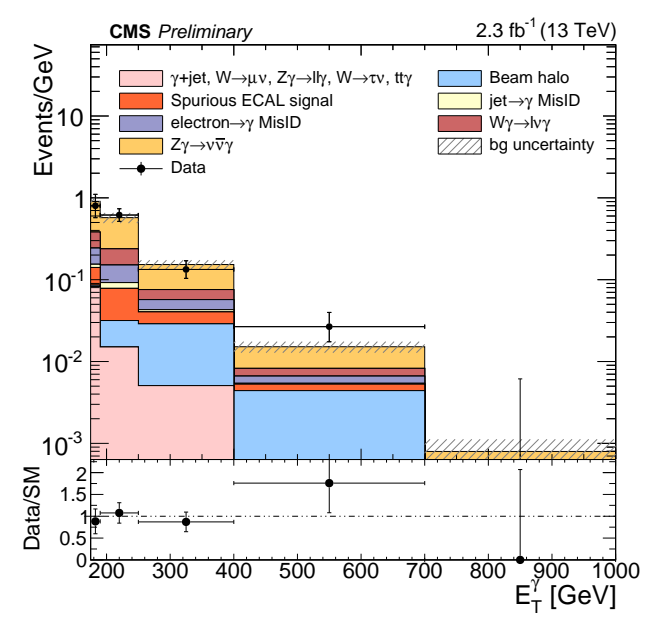

(b)

Figure 1: Distributions of the photon transverse energy for the $8 \mathrm{TeV}$ analysis (a) and $13 \mathrm{TeV}$ analysis (b) of $Z \gamma$ in the $v v \gamma$ final state [5, 6]. The filled histograms represent different predicted contributions to the observed data which are shown as filled points. The hatched regions show the total uncertainty on the sum of all predictions while the error bars on the data points show the statistical error on the observed data. The bottom panels show the ratio of the data to the prediction. In (a) the dashed line shows the predicted distribution for a non-zero value of an anomalous coupling. 


\begin{tabular}{l|c|c}
\hline$\sqrt{s}$ & NNLO Theory (fb) & Measured (fb) \\
\hline $8 \mathrm{TeV}$ & $50.0_{-2.2}^{+2.4}$ & $52.7 \pm 2.1$ (stat.) \pm 6.4 (syst.) \pm 1.4 (lumi.) \\
$13 \mathrm{TeV}$ & $65.5 \pm 3.3$ & $66.5 \pm 13.6$ (stat.) \pm 14.3 (syst.) \pm 2.2 (lumi.)
\end{tabular}

Table 1: Measured and theoretical cross sections for the $8 \mathrm{TeV}$ and $13 \mathrm{TeV}$ results.

Photons are also used to measure vector boson scattering (VBS) in both the $W \gamma$ [7] and $Z \gamma$ [8] final states. In addition to measuring $W \gamma$ and $Z \gamma$ production in unique phase spaces, these analyses also have sensitivity to the pure electroweak components. Events for the $W \gamma$ selection are identified as having single electron or muon, one photon, and two jets having large pseudorapidity separation. The escaping neutrino is identified by requiring $\mathrm{E}_{\mathrm{T}}^{\text {miss }}>35 \mathrm{GeV}$ and transverse mass greater than $30 \mathrm{GeV}$. The $Z \gamma$ selection is similar, but requires two electrons or muons to identify the leptonic $\mathrm{Z}$ boson decay. The $\mathrm{Z}$ boson is identified by requiring the dilepton mass to be $70 \mathrm{GeV}<M_{\ell \ell}<$ $100 \mathrm{GeV}$. Additional event selections are applied to enhance the fraction of VBS events. The dijet system must be separated in $\phi$ from the combined photon and $\mathrm{W}$ or $\mathrm{Z}$ system, the mass of the dijet system $\left(M_{j j}\right)$ must be large , and a cut is applied on the Zeppenfeld variable [9]. The specific cut values are optimized separately for the $W \gamma$ and $Z \gamma$ analyses.

The main backgrounds in determining the electroweak component of both analyses are from the non-VBS continuum production of the same final states. These backgrounds are estimated using Monte Carlo simulated samples which are normalized to control regions where the di-jet invariant mass is less than the signal selection requirement. Systematic uncertainties are assessed for the extrapolation to larger dijet mass using the simulation. The background from jets misidentified as photons is estimated using a data driven method. Smaller backgrounds are estimated using Monte Carlo simulation.

Figure 2 shows the dijet mass distributions for the $W \gamma$ and $Z \gamma$ analyses. The component resulting from the pure electroweak interaction is shown separately. For each analysis the cross section is measured for both the pure electroweak components and the full signal production in the selected phase space. The $W \gamma$ cross section measured in the phase space where $M_{j j}>700 \mathrm{GeV}$ and $\left|\Delta \eta_{j j}\right|>$ 2.4 is $23.2 \pm 4.3$ (stat.) \pm 1.7 (syst.) \pm 0.6 (lumi.) fb. The electroweak component is observed with a significance of $2.7 \sigma$ and the measured cross section is $10.8 \pm 4.1$ (stat.) \pm 3.4 (syst.) \pm 0.3 (lumi.) $\mathrm{fb}$. The $Z \gamma$ cross section measured in the phase space where $M_{j j}>800 \mathrm{GeV}$ and $\left|\Delta \eta_{j j}\right|>2.5$ is $1.00 \pm 0.43$ (stat.) \pm 0.26 (syst.) \pm 0.03 (lumi.) fb. Electroweak production of $Z \gamma$ is observed with a significance of $3.0 \sigma$ in the phase space where $M_{j j}>400 \mathrm{GeV}$ and $\left|\Delta \eta_{j j}\right|>2.5$ and the measured cross section is $1.86_{-0.75}^{+0.89}$ (stat.) ${ }_{-0.27}^{+0.41}$ (syst.) \pm 0.05 (lumi.) fb.

The highest rate of triboson production occurs with photons in the final state. In particular CMS has measured the cross sections of a $\mathrm{W}$ boson produced with two photons and a $\mathrm{Z}$ boson produced with two photons $(W \gamma \gamma, Z \gamma \gamma)$ using $19.4 \mathrm{fb}^{-1}$ of $8 \mathrm{TeV}$ data [10]. The $W \gamma \gamma$ final state involves a quartic gauge coupling and limits are set on aQGC.

The $W \gamma \gamma$ final state is identified by the presence of a muon that passes the trigger and two photons having $p_{T}>25 \mathrm{GeV}$. In addition the magnitude of $\mathrm{E}_{\mathrm{T}}^{\text {miss }}$ is required to be greater than 40 $\mathrm{GeV}$. The $Z \gamma \gamma$ final state is identified by the presence of two electrons or two muons that pass the trigger and two photons having $p_{T}>15 \mathrm{GeV}$. In addition the dilepton invariant mass must be greater than $40 \mathrm{GeV}$. 


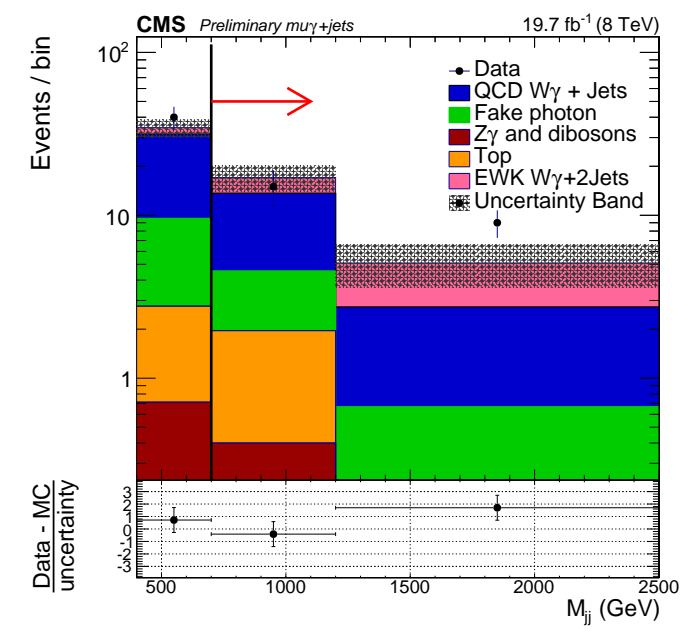

(a)

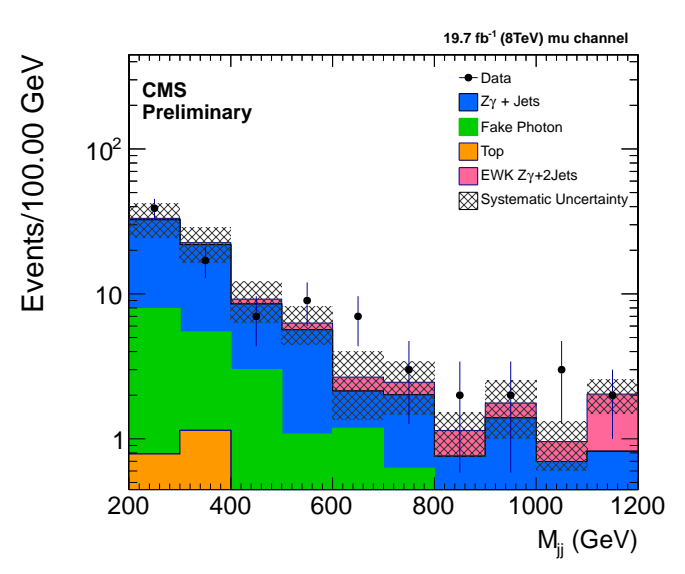

(b)

Figure 2: Distributions of $M_{j j}$ for the $W \gamma$ analysis [7] (a) and $Z \gamma$ analysis [8] (b). The filled histograms show the different predicted contributions to the observed data, which are shown in black points. The component resulting from the pure electroweak interaction is shown separately. The hatched area shows the systematic uncertainty on the sum of the predictions. In (a) the lower panel shows the ratio of the data to the prediction normalized to the uncertainty in the prediction.

The principal background to both analyses is from jets misidentified as photons where either one or both identified photons may result from misidentified jets. A data driven method is employed to estimate this background. The method loosens the isolation requirements on photons and uses templates to describe the four sources of photons ( Prompt-Prompt, Prompt-Fake, Fake-Prompt, Fake-Fake ) that are derived from simulation to describe prompt photons and from data for fake photons. The template normalizations are solved using a matrix equation that inputs the observed data in sidebands having loosened isolation requirements. Remaining backgrounds are estimated using Monte Carlo simulation.

Figure 3 shows the transverse momentum of the diphoton system for the $W \gamma \gamma$ and $Z \gamma \gamma$ analyses. In both cases the signal is clearly observed over the predicted background. The $W \gamma \gamma$ signal is observed with a significance of $2.4 \sigma$ with a measured cross section of $6.0 \pm 1.8$ (stat.) \pm 2.3 (syst.) \pm 0.2(lumi.) fb. In addition limits are placed on anomalous Quartic Gauge Couplings using the phase space where the transverse momentum of the lead photon is greater than $70 \mathrm{GeV}$. The $Z \gamma \gamma$ signal is observed with a significance of $5.9 \sigma$ with a measured cross section of $12.7 \pm 1.4$ (stat.) \pm 1.8 (syst.) \pm 0.3 (lumi.) fb. Both measured cross section are consistent with theoretical predictions.

The CMS experiment has searched for an additional triboson final state, the production of WW and WZ with a photon where one W boson decays leptonically and the second massive boson decays hadronically [11]. The WW and WZ systems are treated as a single final state because the hadronically decaying bosons are not distinguished. A search for this final state was performed using $19.3 \mathrm{fb}^{-1}$ of $8 \mathrm{TeV}$ data. No signal was observed over the background and a limit on the production cross section of 3.4 times the theoretical expectation was placed. In addition, stringent 


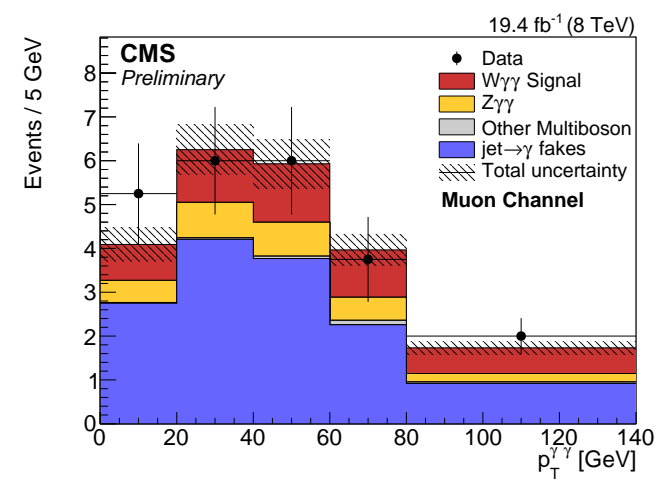

(a)

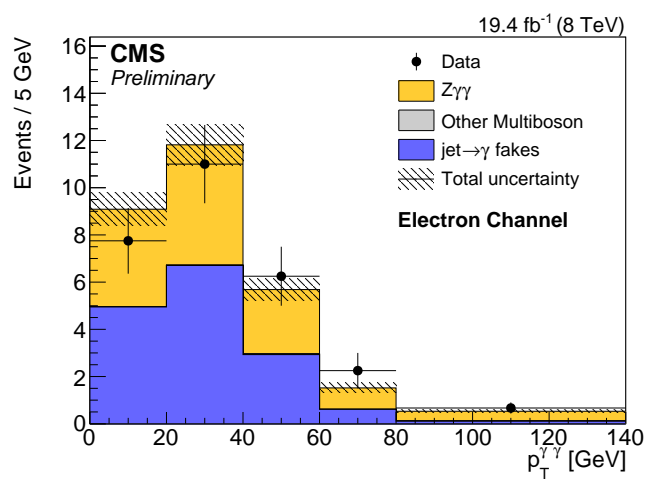

(b)

Figure 3: Distributions of the diphoton transverse momentum for the $W \gamma \gamma$ analysis (a) and $Z \gamma \gamma$ analysis (b) [10]. The filled histograms show the predicted contributions to the observed data which are shown as filled points. The hatched area shows the total uncertainty on the sum of the predictions and the error bars on the data points show the statistical uncertainty on the observed data.

limits were placed on anomalous quartic gauge couplings. Observation of this final state is now possible using the integrated luminosity collected by the LHC during the 2016 run.

\section{Conclusion}

Photons provide unique signatures in multiboson final states at the LHC. In addition to highrate processes such as $\gamma \gamma, W \gamma$ and $Z \gamma$, photons are used to identify rarer processes such as tribosons or processes involving vector boson scattering. The CMS experiment has measured a number of these final states and has used their sensitivity to anomalous gauge couplings to place limits on new physics. A summary of all limits from CMS can be found here ${ }^{2}$. Limits are placed using principally an effective field theory, but also using a vertex formalism for neutral gauge couplings. Triboson and final states involving vector boson scattering provide strong sensitivity to anomalous quartic gauge couplings and therefore provide stringent limits on new physics that may appear in those vertices. In the case where new physics lies just beyond the reach of the LHC such that it cannot be produced resonantly, precise measurements of rare Standard Model processes, such as those presented here, may provide the first hits at its presence.

\section{References}

[1] Celine Degrande, Nicolas Greiner, Wolfgang Kilian, Olivier Mattelaer, Harrison Mebane, Tim Stelzer, Scott Willenbrock, and Cen Zhang. Effective Field Theory: A Modern Approach to Anomalous Couplings. Annals Phys., 335:21-32, 2013.

[2] B. M. Gavela, E. E. Jenkins, A. V. Manohar, and L. Merlo. Analysis of General Power Counting Rules in Effective Field Theory. Eur. Phys. J., C76(9):485, 2016.

\footnotetext{
${ }^{2}$ https://twiki.cern.ch/twiki/bin/view/CMSPublic/PhysicsResultsSMPaTGC
} 
[3] Da Liu, Alex Pomarol, Riccardo Rattazzi, and Francesco Riva. Patterns of Strong Coupling for LHC Searches. 2016.

[4] S. Chatrchyan et al. The CMS experiment at the CERN LHC. JINST, 3:S08004, 2008.

[5] Vardan Khachatryan et al. Measurement of the $Z \gamma \rightarrow v \bar{v} \gamma$ production cross section in pp collisions at $\sqrt{s}=8 \mathrm{TeV}$ and limits on anomalous $\mathrm{ZZ} \gamma$ and $\mathrm{Z} \gamma \gamma$ trilinear gauge boson couplings. Phys. Lett., B760:448-468, 2016.

[6] Measurement of the production cross section for pp to $\mathrm{Z}$ (nu nu) gamma at sqrt(s) $=13 \mathrm{TeV}$ at CMS. Technical Report CMS-PAS-SMP-16-004, CERN, Geneva, 2016.

[7] A search for electroweak-induced production of Wgamma with two jets and constraints on anomalous quartic gauge couplings in pp collisions at sqrt(s) $=8 \mathrm{TeV}$. Technical Report CMS-PAS-SMP-14-011, CERN, Geneva, 1900.

[8] Evidence for the electroweak $\mathrm{Z}$ gamma production in association with two jets and a search for anomalous quartic gauge couplings in pp collisions at sqrts $=8 \mathrm{TeV}$. Technical Report CMS-PAS-SMP-14-018, CERN, Geneva, 2015.

[9] David L. Rainwater, R. Szalapski, and D. Zeppenfeld. Probing color singlet exchange in $Z+$ two jet events at the CERN LHC. Phys. Rev., D54:6680-6689, 1996.

[10] Measurements of the $p p \rightarrow \mathrm{W}^{ \pm} \gamma \gamma$ and $p p \rightarrow \mathrm{Z} \gamma \gamma$ cross sections and Limits on Dimension-8 Effective Anomalous Gauge Couplings at $\sqrt{s}=8 \mathrm{TeV}$. Technical Report CMS-PAS-SMP-15-008, CERN, Geneva, 2016.

[11] Serguei Chatrchyan et al. Search for $W W \gamma$ and $W Z \gamma$ production and constraints on anomalous quartic gauge couplings in $p p$ collisions at $\sqrt{s}=8 \mathrm{TeV}$. Phys. Rev., D90(3):032008, 2014. 